\title{
Uric acid in hypertension - a marker of cardiovascular risk related to body composition
}

\author{
Ewelina Pałkowska-Goździk',2, Paweł Krzesiński' ${ }^{1}$ Katarzyna Piotrowicz' ${ }^{1}$, Adam Stańczyk ${ }^{1}$, \\ Grzegorz Gielerak', Wiesław Piechota ${ }^{3}$, Andrzej Skrobowski ${ }^{1}$ \\ ${ }^{1}$ Department of Cardiology and Internal Diseases, Military Institute of Medicine, Warsaw, Poland \\ ${ }^{2}$ Department of Dietetics, University of Life Sciences-SGGW, Warsaw, Poland \\ ${ }^{3}$ Laboratory Diagnostics Unit, Military Institute of Medicine, Warsaw, Poland
}

Pałkowska E, Krzesiński P, Piotrowicz K, Stańczyk A, Gielerak G, Piechota W, Skrobowski A. Uric acid in hypertension - a marker of cardiovascular risk related to body composition. J Pre-Clin Clin Res. 2015; 9(2): 124-128. doi: 10.5604/18982395.1186492

\begin{abstract}
Introduction. Elevated uric acid (UA) is associated with arterial hypertension (AH), obesity, dyslipidemia and insulin resistance. However, its association with body components has not been previously investigated.

Objective. The aim of this study was to evaluate the relationship between UA and cardiovascular risk factors, anthropometric parameters and body composition in patients with $\mathrm{AH}$.

Materials and method. In 138 patients with $\mathrm{AH}$ the following parameters were evaluated: UA, low and high density lipoproteins (LDL-C, HDL-C), triglycerides (TG), fasting glucose (FG), creatinine; body mass index (BMI), waist circumference (WC), fat mass (FM), fat free mass (FFM) and total body water (TBW).

Results. Positive correlations were shown between UA and LDL-C $(p=0.041)$, TG $(p<0.001), F G(p=0.025)$ and creatinine $(p<0.001)$ and negative between UA and HDL-C $(p<0.001)$. Significant associations between UA and anthropometric parameters and body components, such as WC $(p<0.001), B M I(p<0.001)$, FFM $(p<0.001)$ and TBW $(p<0.001)$, were also observed. In the multiple regression model, independent predictors of UA concentration were serum creatinine and TBW $\left(R^{2}=0.45 ; p<0.001\right)$.

Conclusions. In patients with $\mathrm{AH}$, uric acid was significantly related to cardiovascular risk factors, including obesity. However, the main anthropometric determinant of plasma UA concentration is FFM. The consideration of body composition in the interpretation of UA concentration appears to be justified, but the verification of this hypothesis requires further studies.
\end{abstract}

\section{Key words}

hypertension, risk factors, visceral obesity, hyperuricaemia

\section{INTRODUCTION}

Arterial hypertension ( $\mathrm{AH}$ ) is one of the most prevalent chronic disease which every year causes almost 7.5 million deaths worldwide [1]. One of the metabolic risk factors related to $\mathrm{AH}$ is increased uric acid (UA) concentration [2, 3]. Hyperurycaemia proved to be especially associated with incidents of myocardial infarction and stroke $[4,5]$. The prevalence of increased UA is estimated to affect about 25$40 \%$ hypertensive patients, while almost $50-70 \%$ patients with hyperurycaemia present abnormal blood pressure (BP) 6].

Increased UA concentration is usually recognized as a negative risk factor [5]. Hyperurycaemia proved to be associated with metabolic disturbances, i.e. dyslipidaemia, insulin resistance and obesity [7, 8, 9]. It is believed that in mild and moderate $\mathrm{AH}$, increased UA concentration is mostly the effect of impaired renal excretion [10]. It was also observed that UA participated in the complex activity of fat tissue, oxidative stress, pro-inflammatory activity and water-electrolyte balance $[3,10,11,12]$. On the other hand, UA presents some antioxidative properties and may be associated with the improvement of cardiac performance in heart failure [13] and lower risk of neurodegenerative diseases and sarcopenia in elderly patients [14].

Address for correspondence: Ewelina Pałkowska, Department of Cardiology and Internal Diseases, Military Institute of Medicine, Szaserów 128, 04-441 Warsaw, Nowoursynowska 159c, 02-776 Warsaw, Poland

E-mail: ewelina.palkowska@poczta.onet.pl

Received: 09 June 2015; accepted: 27 October 2015
Thus, the interpretation of the association between UA and 'obesity', defined by means of the classic parameters, such as waist circumference (WC) and body mass index (BMI), should take into consideration the fact that they reflect the body composition and proportion between fat and free fat mass imperfectly $[10,15]$.

The presented study hypothesizes that evaluation of the relationship between UA concentration, cardiovascular risk (CVR) factors and body composition in patients with unmedicated $\mathrm{AH}$ would reveal some clinically important pathophysiological features addressing the true origin of the increased UA concentration.

\section{OBJECTIVE}

Within the above context, the aim of the study was to evaluate the relationship between UA concentration and cardiovascular risk factors, anthropometric parameters and body composition in patients with $\mathrm{AH}$.

\section{MATERIALS AND METHOD}

Study population. The analysis comprised 138 patients with at least a 3-month history of $\mathrm{AH}$, defined according to the European Society of Cardiology Guidelines [16]. The diagnosis of $\mathrm{AH}$ was verified with the use of ambulatory blood pressure monitoring. Patients treated with hypotensive 
drugs were recommended to discontinue them at least 7 days before the examination.

Exclusion criteria. 1) confirmed secondary AH; 2) $\mathrm{AH}$ treated with 3 or more medicines before recruitment; 3 ) heart failure; 4) cardiomyopathy; 5) significant heart rhythm disorders; 6) significant valvular disease; 7) kidney failure (GFR below $60 \mathrm{ml} / \mathrm{min}^{\star} 1.73 \mathrm{~m}^{2}$ ); 8) chronic obstructive pulmonary disease; 9) diabetes; 10) polyneuropathy; 11) peripheral vascular disease; 12) age $<18$ years.

The group selected for this retrospective analysis comprised patients from a clinical study registered at ClinicalTrials.gov (NCT01996085). The study was conducted according to the Good Clinical Practice Guidelines and the Declaration of Helsinki, with the approval of the Local Ethics Committee. Each patient provided informed written consent to participate in the study.

Clinical examination. A clinical examination was performed with consideration of CVR factors and symptoms indicating a secondary cause of $\mathrm{AH}$ [16]. Anthropometric characteristics included: height, weight, WC and BMI. The office blood pressure measurement was performed automatically (Omron M4 Plus, Japan) by a technique in compliance with the European Society of Cardiology Guidelines. Systolic blood pressure (SBP) and diastolic blood pressure (DBP) were measured in a quiet room, in the presence of a trained physician or nurse, and after a minimum of 5 minutes resting in a sitting position. The subjects were seated comfortably with an arm supported and legs uncrossed. The BP category of $\mathrm{AH}$ was defined according to the European Society of Cardiology Guidelines [16]: grade 1 - SBP 140-159 mmHg and/or DBP 90-99 mmHg, grade 2 - SBP 160-179 mmHg and/or DBP 100-109 mmHg, grade 3-SBP greater than 180 $\mathrm{mmHg}$ and/or DBP greater than $110 \mathrm{mmHg}$.

Laboratory tests. Laboratory tests included the evaluation of renal function (creatinine, urea, GFR - glomerular filtration rate) and metabolic disturbances: fasting glucose (FG), total cholesterol (TC), low density lipoproteins (LDL-C) high density lipoproteins (HDL-C), triglycerides (TG) and uric acid (UA). The criteria of IDF were applied to identify patients with metabolic syndrome (MS) [17].

Body composition assessment. Body composition assessment was performed using bioelectrical impedance analysis (Tanita BC 418 MA analyzer, Tanita Corporation, Japan) in the early morning hours, about 2-4 hours after a light breakfast. The assessment included evaluation of: body mass, fat mass and its percentage (FM, \%FM), fat free mass (FFM, \%FFM) and total body water (TBW).

Cardiovascular risk assessment. Cardiovascular risk assessment recommended by the European Society of Cardiology (ESC) Guidelines for the management of AH [16] was applied. Stratification of total CVR was performed the in categories of: 'low', 'moderate', 'moderate/high', 'high' and 'very high' risk, according to SBP and DBP and prevalence of risk factors such as: gender (male); age $>55$ years (for females) and $>65$ years (for males); obesity $\left(\mathrm{BMI}>30 \mathrm{~kg} / \mathrm{m}^{2}\right.$ ); central obesity - WC $>88 \mathrm{~cm}$ (for females) and WC>102 cm (for males); current smoking; family history of premature cardiovascular diseases (at age $<65$ years for females and
$<55$ years for males); dyslipidemia (TC $>190 \mathrm{mg} / \mathrm{dl}$ and/or LDL-C $>115 \mathrm{mg} / \mathrm{dl}$ and/or HDL-C $<46 \mathrm{mg} / \mathrm{dl}$ for females and $<40 \mathrm{mg} / \mathrm{dl}$ for males and/or TG >150 mg/dl); FG > $102 \mathrm{mg} / \mathrm{dl}$.

Statistical analysis. Statistical analysis was performed using Statistica 7.0 (StatSoft, Inc.). Distribution and normality of the data were assessed by visual inspection and the KolmogorovSmirnov test. Continuous variables were presented as means \pm standard deviations (SD), and categorical variables as absolute values and relative frequencies (percentages). To analyze the differences in UA between subgroups of different CVR, ANOVA (analysis of variance) was used (detailed intersubgroups comparison was performed using the adequate post-hoc test). Relationships between UA and other analyzed parameters were investigated with Pearson's (Spearman's) correlation coefficients. The most representative variables were included in the multivariate regression model. A p value of $<0.05$ was taken to indicate statistical significance.

\section{RESULTS}

Characteristics of the patients in the study group are shown in Table 1, dominated by those with mild hypertension, among whom more than a half presented co-existing metabolic disturbances. The total CVR of almost $75 \%$ of subjects was classified as 'moderate/high' and 'high'. No patient was

Table 1. Basic characteristics of study group

\begin{tabular}{|c|c|}
\hline Parameters & $\begin{array}{l}\text { Study group } \\
\qquad(\mathrm{n}=138)\end{array}$ \\
\hline Men, n (\%) & $95(68.8)$ \\
\hline age (years), mean \pm SD & $44.9 \pm 10.4$ \\
\hline age > 55 years (women) and > 65 years (men) , n (\%) & $15(10.9)$ \\
\hline $\mathrm{SBP}(\mathrm{mmHg})$, mean $\pm \mathrm{SD}$ & $141.0 \pm 13.0$ \\
\hline $\mathrm{DBP}(\mathrm{mmHg})$, mean $\pm \mathrm{SD}$ & $90.3 \pm 9.4$ \\
\hline $\mathrm{AH}$ - grade $1, \mathrm{n}(\%)$ & $112(91.2)$ \\
\hline $\mathrm{AH}$ - grade $2, \mathrm{n}(\%)$ & $26(18.8)$ \\
\hline weight (kg), n (\%) & $88.1 \pm 16.2$ \\
\hline height (m), n (\%) & $173.7 \pm 9.9$ \\
\hline $\mathrm{BMI}\left(\mathrm{kg} / \mathrm{m}^{2}\right)$, mean $\pm \mathrm{SD}$ & $29.1 \pm 4.3$ \\
\hline$W C(\mathrm{~cm})$, mean $\pm S D$ & $99.1 \pm 11.9$ \\
\hline family history of premature cardiovascular disease, $\mathrm{n}(\%)$ & $47(34.1)$ \\
\hline family history of $\mathrm{AH}, \mathrm{n}(\%)$ & $99(71.7)$ \\
\hline family history of ischemic heart disease, n (\%) & $52(37.7)$ \\
\hline previous hypotensive treatment, $\mathrm{n}(\%)$ & $28(20.3)$ \\
\hline $\mathrm{FG}(\mathrm{mg} / \mathrm{dl})$, mean $\pm \mathrm{SD}$ & $98.2 \pm 11.3$ \\
\hline $\mathrm{TC}(\mathrm{mg} / \mathrm{dl})$, mean $\pm \mathrm{SD}$ & $224.6 \pm 40.0$ \\
\hline $\mathrm{TG}(\mathrm{mg} / \mathrm{dl})$, mean $\pm \mathrm{SD}$ & $157.6 \pm 78.9$ \\
\hline $\mathrm{LDL}-\mathrm{C}(\mathrm{mg} / \mathrm{dl})$, mean $\pm \mathrm{SD}$ & $146.0 \pm 34.9$ \\
\hline $\mathrm{HDL}-\mathrm{C}(\mathrm{mg} / \mathrm{dl})$, mean $\pm \mathrm{SD}$ & $57.7 \pm 18.5$ \\
\hline Creatinine $(\mathrm{mg} / \mathrm{dl})$, mean $\pm \mathrm{SD}$ & $0.84 \pm 0.16$ \\
\hline $\mathrm{GFR}\left(\mathrm{ml} / \mathrm{min} / 1.73 \mathrm{~m}^{2}\right)$, mean $\pm \mathrm{SD}$ & $100.1 \pm 18.8$ \\
\hline $\mathrm{UA}(\mathrm{mg} / \mathrm{dl})$, mean $\pm \mathrm{SD}$ & $5.98 \pm 1.28$ \\
\hline
\end{tabular}

$\mathrm{AH}$ - arterial hypertension; BMI - body mass index; DBP - diastolic blood pressure; GFR glomerular filtration rate; HDL-C - high density lipoproteins;

FG-fasting glucose; LDL-C - low density lipoproteins; SBP - systolic blood pressure; SD - standard deviation; TC - total cholesterol; TG - triglycerides; UA - uric acid; deviation; TC - total choles
WC - waist circumference; 
categorized as 'very high' CVR (Fig. 1). A combined increase of UA concentration and CVR was observed (Tab. 2).

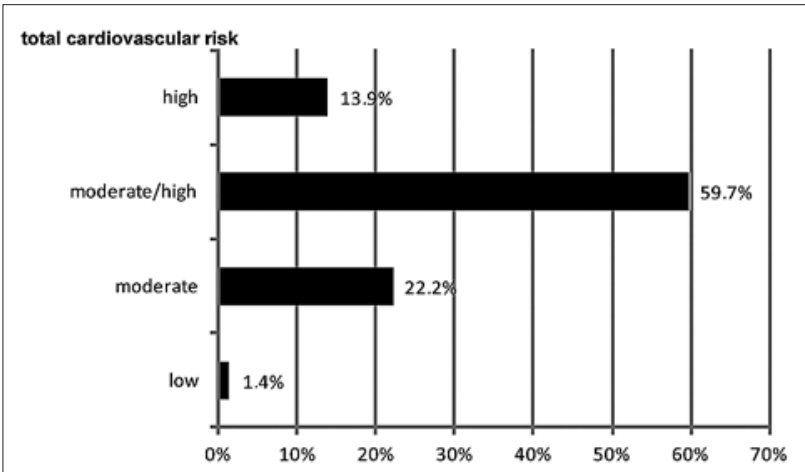

Figure 1. Distribution of categories of cardiovascular risk in the study group

Table 2. Uric acid concentration and total cardiovascular risk categories

\begin{tabular}{|c|c|c|c|c|}
\hline \multicolumn{4}{|c|}{ Total cardiovascular risk } & \multirow[t]{2}{*}{$\mathrm{p}$} \\
\hline low $(n=2)$ & $\begin{array}{c}\text { moderate } \\
(n=30)\end{array}$ & $\begin{array}{c}\text { moderate/high } \\
\qquad(\mathrm{n}=86)\end{array}$ & High $(n=20)$ & \\
\hline \multicolumn{5}{|c|}{ uric acid, mg/dl } \\
\hline $4.80 \pm 0.87$ & $5.24 \pm 0.22$ & $6.18 \pm 0.13^{* *}$ & $6.33 \pm 0.27^{*}$ & 0.001 \\
\hline \multicolumn{2}{|c|}{$5.22 \pm 1.15$} & \multicolumn{2}{|c|}{$6.20 \pm 1.24$} & 0.00009 \\
\hline
\end{tabular}

** - statistically significant difference between subgroups of moderate and moderate/high cardiovascular risk: $\mathrm{p}<0.01$

* - statistically significant difference between subgroups of moderate and moderate and high cardiovascular risk: $\mathrm{p}<0.05$

Table 3. Correlations between uric acid concentration and clinical, biochemical and haemodynamic parameters

\begin{tabular}{lcc}
\hline vs. UA & $\mathrm{r}$ & $\mathrm{p}$ \\
\hline age & -0.07 & 0.514 \\
\hline SBP & 0.10 & 0.232 \\
\hline DBP & 0.10 & 0.222 \\
\hline height & 0.47 & $<0.001$ \\
\hline weight & 0.53 & $<0.001$ \\
\hline BMI & 0.30 & $<0.001$ \\
\hline WC & 0.53 & $<0.001$ \\
\hline FM & 0.08 & 0.375 \\
\hline \%FM & -0.23 & 0.008 \\
\hline FFM & 0.60 & $<0.001$ \\
\hline \% FFM & 0.22 & 0.011 \\
\hline TBW & 0.61 & $<0.001$ \\
\hline FG & 0.19 & 0.025 \\
\hline TC & 0.14 & 0.108 \\
\hline LDL-C & 0.18 & 0.041 \\
\hline HDL-C & -0.39 & $<0.001$ \\
\hline TG & 0.46 & $<0.001$ \\
\hline GFR & -0.30 & $<0.001$ \\
\hline Creatinine & 0.61 & $<0.001$ \\
\hline BM & &
\end{tabular}

BMI - body mass index; DBP - diastolic blood pressure; GFR - glomerular filtration rate; HDL-C - high density lipoproteins; FG - fasting glucose; FFM, \%FFM - fat free mass and its percentage: FM, \%FM - fat mass and its percentage; LDL-C - low density lipoproteins; R - correlation coefficient; SBP - systolic blood pressure; TBW - and total body water; TC - total cholesterol; TG - triglycerides; UA - uric acid, WC - waist circumference
Relations between uric acid concentration and clinical, biochemical and haemodynamic parameters. Uric acid concentration was positively correlated with BMI, WC, FFM, $\%$ FFM and TBW, and negatively with \%FM. Higher UA was also associated with higher creatinine concentration and metabolic disturbances (higher FG, LDL-C, TG and lower HDL-C) (Tab. 3; Fig. 2). No significant correlation was observed between UA and age, BP and TC.

In the multivariate regression model, including the most representative variables (gender, creatinine, FM, FFM, TBW), creatinine and TBW revealed to be the independent
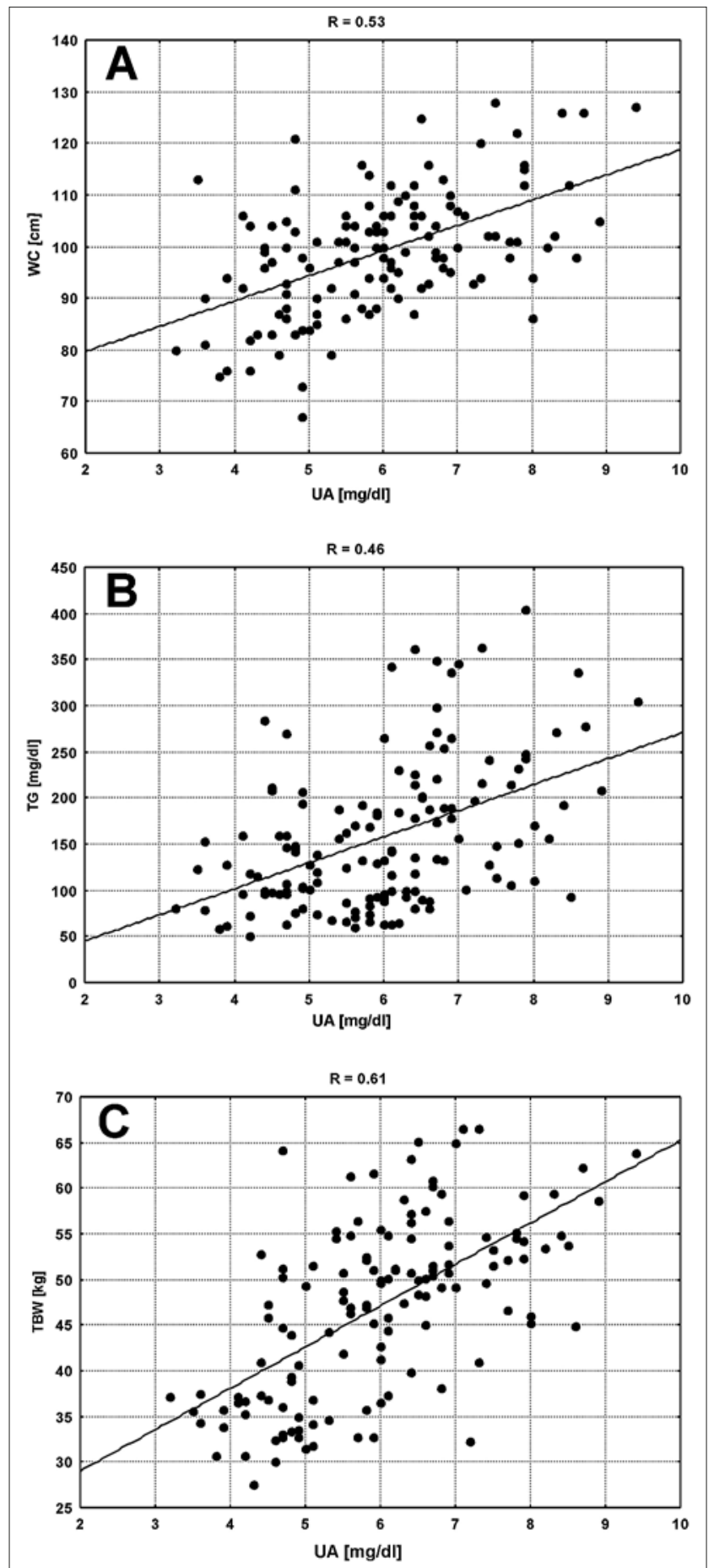

Figure 2. Correlation charts.

(A) uric acid (UA) vs. waist circumference (WC); (B) uric acid (UA) vs. triglycerides (TG); (B) uric acid (UA) vs. total body water (TBW) 
predictors of UA concentration (Rsquare for the model 0.45 ; $\mathrm{p}<0.001)$.

\section{DISCUSSION}

The results of the presented study clearly confirm that UA concentration is associated with metabolic CVR factors and renal function. Strong correlations were also noted between UA concentration and anthropometric indices. In several previous studies $[10,18,19]$, the authors also demonstrated a relationship between high UA concentration and obesity. However, detailed analysis revealed that UA concentration is not directly dependent on FM, but mainly on FFM and TBW.

Hyperuricaemia is commonly associated with traditional cardiometabolic risk factors, such as: glucose intolerance, dyslipidaemia, central obesity and abnormal BP [5]. Since it has been suggested that UA may simply be a consequence of its lower renal excretion related to hyperinsulinaemia, there is growing data that elevated UA concentration may be a predictor of metabolic syndrome, cardiovascular diseases and diabetes [8]. Facchini et al. [20] reported that UA turnover strongly depends on the insulin resistance. A meta-analysis of data from 11 cohort studies indicated hyperuricaemia as a predictor of the occurrence of diabetes [21]. It was observed that each excessive $1 \mathrm{mg} / \mathrm{dl}$ of UA increases diabetes risk by about $17 \%$ [21]. That strong relation between UA and TG that was found also in the presented study has been previously demonstrated $[18,22]$. De Oliveira et al. [18] noted that individuals with a demonstrated increase in TG concentration showed an approximately 2.5 -fold greater risk of hyperuricaemia, regardless of other variables included in MS. According to Matsuura et al. [23], excessive hepatic free fatty acid synthesis in hypertriglyceridaemia accelerates purine synthesis de novo, which in turn increases the production of UA. The negative correlation between UA and $\mathrm{HDL}-\mathrm{C}$ concentrations in the presented study was also previously described [7], and is supposed to be related to oxidative stress and inflammation $[24,25]$.

Positive correlations between UA concentration, BMI and $\mathrm{WC}$, which were noted in the current study, are also well documented $[19,26]$. The stronger association with WC than BMI agrees with the observations indicating greater impact of visceral than subcutaneous fat mass on UA concentration $[19,27]$. Waist circumference is regarded as a reliable indicator of metabolic activity of visceral adipose tissue which significantly correlates with cardiometabolic risk factors, regardless of the amount of subcutaneous fat [15].

Detailed analysis revealed some new insights into the relationship between UA and body composition, indistinguishable with the use of classic anthropometric parameters (WC, BMI). In the current study, it was found that the relationship between UA and both TBW and FFM was stronger than with FM. The most convincing explanation for this observation seems to be the dependence between UA and the skeletal muscle mass, which is indirectly reflected in FFM and TBW $[28,29]$. That observation was supported by a significant correlation noted between UA and creatinine, the concentration of which is strongly connected with muscle mass [30].

To-date, the relationship between muscle mass and UA concentration has not been taken into consideration in common clinical practice. However, the presented results encourage interpreting UA concentration with regard to body composition (especially skeletal muscle mass). It may be supposed that a reduction in GFR may be another issue in the relationship between UA and FFM/TBW [31]. Subclinical accumulation of extracellular water in obese individuals may be caused by chronic inflammation, oxidative stress and endothelial dysfunction [32]. Thus, the true origin of UA concentration in hypertensives is complex and should be investigated in larger study groups.

Limitations. A limitation of this study was the nonrandom selection of the study group, which was dominated by residents of a large urban agglomeration. Although bioelectrical impedance analysis is the best method for estimating body composition in clinical practice, it allows only indirect evaluation of the muscle mass. The authors also realise that the presented results refer to the subjects with $\mathrm{AH}$ without other comorbidities, which limits their extrapolation to the general population.

\section{CONCLUSIONS}

Uric acid is significantly related to cardiovascular risk factors in patients with $\mathrm{AH}$, including abdominal obesity. However, the main anthropometric determinant of plasma UA concentration is FFM. The consideration for body composition in the interpretation of UA concentration appears to be justified, but verification of this hypothesis requires further studies.

\section{Acknowledgements}

The authors erxpress their thanks to the medical staff of the Department of Cardiology and Internal Diseases of the Military Institute of Medicine, especially Drs: Robert Wierzbowski, Beata Uziębło-Życzkowska, Małgorzata Kurpaska, Katarzyna Hałas, Magdalena Potapowicz-Krysztofiak, Agnieszka Jaguś-Jamioła, Łukasz Michalczyk, Agnieszka Wójcik, Anna Kazimierczak, Agnieszka Jurek, Kalina Wolszczak, Agata Galas, for assistance in patient care and data collection, nurses Lidia Wojda and Lidia Latosek for nursing care and data collection. The study was performed as part of the statutory project of the Ministry of Science and Higher Education and Military Institute of Medicine (ID 335), Warsaw, Poland..

\section{REFERENCES}

1. World Health Organization. Global Health Risks: Mortality and Burden of Disease Attributable to Selected Major Risks. Geneva: World Health Organization; 2010; 11.

2. Grayson PC, Kim SY, LaValley M, Choi HK. Hyperuricemia and incident hypertension: a systematic review and meta-analysis. Arthritis Care Res. (Hoboken) 2011; 63: 102-110.

3. Turak O, Ozcan F, Tok D, Işleyen A, Sökmen E, Taşoğlu I, et al. Serum uric acid, inflammation and nondipping circadian pattern in essential hypertension. J Clin Hypertens. (Greenwich) 2013; 15: 7-13.

4. Bos MJ, Koudstaal PJ, Hofman A, Witteman JC, Breteler MM. Uric acid is a risk factor for myocardial infarction and stroke - The Rotterdam Study. Stroke 2006; 37: 1503-1507.

5. Kim SY, Guevara JP, Kim KM, Choi HK, Heitjan DF, Albert DA. Hyperuricemia and Coronary Heart Disease: A Systematic Review and Meta-Analysis. Arthritis Care Res. (Hoboken) 2010; 62: 170-180.

6. Sui X, Church TS, Meriwether RA, Lobelo F, Blair SN. Uric acid and the development of metabolic syndrome in women and men. Metabol Clin Exp. 2008; 57: 845-852. 
7.Lippi G, Montagnana M, Luca Salvagno G, Targher G, Cesare Guidi G. Epidemiological association between uric acid concentration in plasma, lipoprotein(a), and the traditional lipid profile. Clin Cardiol. 2010; 33: 76-80.

8. Soltani Z, Rasheed K, Kapusta DR, Reisin E. Potential role of uric acid in metabolic syndrome, hypertension, kidney injury, and cardiovascular diseases: is it time for reappraisal? Curr Hypertens Rep.2013; 15: 175-181

9. Tykarski A. [The mechanism of hyperuricemia and the assessment of the impact of antihypertensive drugs on the transport of uric acid and its precursors in nehron in primary hypertension.] Habilitation thesis. Poznan University of Medical Sciences, Poznan; 1997.

10. Kim TH, Lee SS, Yoo JH, Kim SR, Yoo SJ, Song HC, et al. The relationship between the regional abdominal adipose tissue distribution and the serum uric acid levels in people with type 2 diabetes mellitus. Diabetol Metab Syndr. 2012; 4: 3.

11. Cicero AF, Rosticci M, Cagnati M, Urso R, Scapagnini G, Morbini M, et al. Brisighella Heart Study Group. Serum uric acid and markers of lowdensity lipoprotein oxidation in nonsmoking healthy subjects: data from the Brisighella Heart Study. Pol Arch Med Wewn. 2014; 124: 661-668.

12. Frey FJ. Serum concentration of uric acid, a diagnostic 'must' in patients with hyponatremia. Ther Umsch. 2004; 61: 583-587.

13. Reyes AJ. The increase in serum uric acid concentration caused by diuretics might be beneficial in heart failure. Eur J Heart Fail. 2005; 7: 461-467.

14. Wu Y, Zhang D, Pang Z, Jiang W, Wang S, Tan Q. Association of serum uric acid level with muscle strength and cognitive function among Chinese aged 50-74 years. Geriatr Gerontol Int. 2013; 13: 672-677.

15. Li X, Katashima M, Yasumasu T, Li KJ. Visceral fat area, waist circumference and metabolic risk factors in abdominally obese Chinese adults. Biomed Environ Sci. 2012; 25: 141-148.

16. Mancia G, Fagard R, Narkiewicz K, Redón J, Zanchetti A, Böhm M, et al. $2013 \mathrm{ESH} / \mathrm{ESC}$ Guidelines for the management of arterial hypertension: the Task Force for the management of arterial hypertension of the European Society of Hypertension (ESH) and of the European Society of Cardiology (ESC). J Hypertens. 2013; 31: 1281-1357.

17. Alberti KG, Zimmet P, Shaw J, IDF Epidemiology Task Force Consensus Group. The metabolic syndrome: a new worldwide definition. Lancet 2005; 366: 1059-1062.

18. de Oliveira EP, Moreto F, Silveira LV, Burini RC. Dietary, anthropometric, and biochemical determinants of uric acid in free-living adults. Nutr J. 2013; 12: 11 .

19. Tsushima Y, Nishizawa H, Tochino Y, Nakatsuji H, Sekimoto R, Nagao $\mathrm{H}$, et al. Uric acid secretion from adipose tissue and its increase in obesity. J Biol Chem. 2013; 288: 27138-27149.

20. Facchini F, Chen YD, Hollenbeck CB, Reaven GM. Relationship between resistance to insulin-mediated glucose uptake, urinary uric acid clearance, and plasma uric acid concentration. JAMA 1991; 266 3008-3011.

21. Kodama S, Saito K, Yachi Y, Asumi M, Sugawara A, Totsuka K, et al. Association between serum uric acid and development of type 2 diabetes. Diabetes Care 2009; 32: 1737-1742.

22.Zhao LJ, Zhao D, Liu J, Wang W, Wu GX, Qin LP, Liu J, et al. Association between serum uric acid and triglyceride in a Chinese community. Zhonghua Nei Ke Za Zhi. 2005; 44: 664-667.

23. Matsuura F, Yamashita S, Nakamura T, Nishida M, Nozaki S, Funahashi $\mathrm{T}$, et al. Effect of visceral fat accumulation on uric acid metabolism in male obese subjects: visceral fat obesity is linked more closely to overproduction of uric acid than subcutaneous fat obesity. Metabolism 1998; 47: 929-933.

24. Vekic J, Jelic-Ivanovic Z, Spasojevic-Kalimanovska V, Memon L, Zeljkovic A, Bogavac-Stanojevic N, et al. High serum uric acid and low-grade inflammation are associated with smaller LDL and HDL particles. Atherosclerosis 2009; 203: 236-242.

25. Karabacak M, Varol E, Kahraman F, Ozaydin M, Türkdogan AK, Ersoy IH. Low high-density lipoprotein cholesterol is characterized by elevated oxidative stress. Angiology 2014; 65: 927-931.

26. Hikita M, Ohno I, Mori Y, Ichida K, Yokose T, Hosoya T. Relationship between hyperuricemia and body fat distribution. Intern Med. 2007; 46: $1353-1358$.

27. Tamba S, Nishizawa H, Funahashi T, Okauchi Y, Ogawa T, Noguchi $\mathrm{M}$, et al. Relationship between the serum uric acid level, visceral fat accumulation and serum adiponectin concentration in Japanese men. Intern Med. 2008; 47: 1175-1180.

28. Kinugawa T, Ogino K, Kato M, Kato T, Osaki S, Endo A, et al. Altered purine and glycogen metabolism in skeletal muscle during exercise in patients with heart failure. Metabolism. 1999; 48: 484-488.

29. Ellegård L, Tengvall M. Bioelectrical Impedance to Predict Muscle Mass in the Elderly. In: Preedy VR, eds. Handbook of Anthropometry: Physical Measures of Human Form in Health and Disease. Springer New York: Dordrecht Heidelberg; 2012; 375-385.

30. Baxmann AC, Ahmed MS, Marques NC, Menon VB, Pereira AB, Kirsztajn GM, et al. Influence of muscle mass and physical activity on serum and urinary creatinine and serum cystatin C. Clin J Am Soc Nephrol. 2008; 3: 348-354.

31. Satirapoj B, Supasyndh O, Nata N, Phulsuksombuti D, Utennam D, Kanjanakul I, et al. High levels of uric acid correlate with decline of glomerular filtration rate in chronic kidney disease. J Med Assoc Thai 2010; 93: 65-70.

32.Zoccali C, Mallamaci F. Uric acid, hypertension, and cardiovascular and renal complications. Curr Hypertens Rep. 2013; 15: 531-537. 